\title{
Interstitial lung disease in an adolescent child associated with a novel STAT5B mutation-a case report
}

\author{
Samriti Gupta ${ }^{1}$, Anita Mandal ${ }^{1}$, Kana $\mathrm{Jat}^{1}$, and Sushil Kabra ${ }^{1}$ \\ ${ }^{1}$ All India Institute of Medical Sciences
}

September 25, 2021

\begin{abstract}
STAT5B protein is an important component of signalling pathway for growth hormone and IL-2 mediated responses. Mutation in STAT5B gene has been reported to be associated with growth hormone insensitivity, immunodeficiency and autoimmunity. Chronic pulmonary involvement is also one component of the disorder. We describe a case an adolescent girl who suffered with poor growth and loose stools since 1 year of age and was subsequently diagnosed with celiac disease and hypothyroidism. She developed features of interstitial lung disease (ILD) which were progressively worsening. Common causes of ILD were ruled out on investigations. She underwent whole exome sequencing and found to be positive for a novel mutation in STAT5B gene on exon 16. On further evaluation, she had growth hormone insensitivity and CD8 cell deficiency. This case highlights that in a child with chronic pulmonary involvement with features suggestive of growth hormone insensitivity, immunodeficiency and autoimmunity, evaluation for STAT5B mutation should be thought of.
\end{abstract}

\section{Interstitial lung disease in an adolescent child associated with a novel STAT5B mutation-a case report}

Samriti Gupta, MD, DM; Anita Mandal, MD; Kana Ram Jat, MD; Sushil Kumar Kabra, MD

Affiliation: Department of Pediatrics, All India Institute of Medical Sciences, New Delhi, India

Corresponding Author: Sushil Kumar Kabra,

Professor, Division of Pediatric pulmonology and intensive care, Department of Pediatrics, All India Institute of Medical Sciences, New Delhi, India skkabra@hotmail.com

Conflict of Interest: None

Funding: None

Keywords: STAT5B mutation; interstitial lung disease; child; growth hormone insensitivity; immunodeficiency

Running head: A novel STAT5B mutation as a cause of ILD

\section{Abstract}

STAT5B protein is an important component of signalling pathway for growth hormone and IL-2 mediated responses. Mutation in STAT5B gene has been reported to be associated with growth hormone insensitivity, immunodeficiency and autoimmunity. Chronic pulmonary involvement is also one component of the disorder. We describe a case an adolescent girl who suffered with poor growth and loose stools since 1 year of age and was subsequently diagnosed with celiac disease and hypothyroidism. She developed features of interstitial lung disease (ILD) which were progressively worsening. Common causes of ILD were ruled out on investigations. She underwent whole exome sequencing and found to be positive for a novel mutation in STAT5B gene 
on exon 16. On further evaluation, she had growth hormone insensitivity and CD8 cell deficiency. This case highlights that in a child with chronic pulmonary involvement with features suggestive of growth hormone insensitivity, immunodeficiency and autoimmunity, evaluation for STAT5B mutation should be thought of.

\section{Background}

Signal transduction and activation of transcription 5B (STAT5B) protein is involved in signal transduction of growth hormone $(\mathrm{GH})$ receptor and mediates actions of IL-2 and hence, plays an essential role in growth and immunity. Mutations in STAT5B gene are associated with growth failure, immunodeficiency, autoimmunity and diffuse lung disease (DLD) (1).

Lung involvement in STAT5B mutations or deficiency has been reported predominantly because of recurrent pulmonary infections or lymphoid interstitial pneumonitis (LIP) (2). We report a case of an adolescent girl with a novel mutation in STAT5B gene who presented with DLD along with autoimmunity (celiac disease), CD8 cell deficiency and GH insensitivity (GHI).

\section{Case report}

An 11 years old female was born out of non-consanguineous marriage with normal birth weight $(3.2 \mathrm{~kg})$ and length $(51 \mathrm{~cm})$. She was growing well till 1 year of age when she developed recurrent loose stools and had poor weight gain. At 15 months of age, in view of poor weight gain and bilateral infiltrates on chest $\mathrm{X}$ ray (CXR), anti-tubercular treatment was given for 6 months but she had no response. At 5 years of age, she was diagnosed to have celiac disease with anti-tTG IgA levels- $300 \mathrm{U} / \mathrm{ml}$ (normal value $<15 \mathrm{U} / \mathrm{ml}$ ) and hypothyroidism (TSH-52.3 mIU/L) (normal value 0.5-5.5 mIU/L). She was started on gluten free diet and L-thyroxine and had shown symptomatic improvement with some weight gain. At 8 years of age, she developed insidious onset fast breathing which gradually progressed from MRC grade 1 to 4 over 3 years along with dry, non-productive cough. She had minimal response to bronchodilators and inhaled steroids. She underwent CT Chest which was reported as having fibrotic changes. There were no similar family history or any sibling death. There was history of exposure to pigeons. In view of no response in respiratory symptoms and further evaluation, she was referred to our centre.

At presentation to our centre, she had tachypnea and respiratory distress with normal oxygen saturation, grade 3 clubbing and growth failure [weight $19.3 \mathrm{~kg}(-2.83 \mathrm{Z}$ score), height $122 \mathrm{~cm}(-2.88 \mathrm{Z}$ score), body mass index (BMI) 12.96 (-1.88 Z score)]. She was evaluated for various causes of childhood interstitial lung disease (ILD) mainly autoimmune diseases, hypersensitivity pneumonitis and NKX2.1 mutation.

Complete hemogram and metabolic panel were within normal limits. CXR revealed bilateral reticulonodular shadows in all lung fields. CT chest showed organizing pneumonia in right upper lobe. Other lobes showed septal thickening and fibrotic changes (Fig 1). Spirometry was suggestive of severe restrictive disease. Autoimmune antibody panel were negative. Bronchoalveolar lavage (BAL) CD4:CD8 ratio (0.79) was reduced. Serum precipitins for avian antigen were negative. Lung biopsy couldn't be done due to refusal by parents. Echocardiography showed features suggestive of mild pulmonary hypertension. Whole exome sequencing revealed a novel mutation in STAT5B gene which was a homozygous missense variation in exon $16(\operatorname{chr} 17: 40359647 \mathrm{~A}>\mathrm{C}$; c.2006T $>\mathrm{G})$ that results in the amino acid substitution of Glycine for Valine at codon 669 (p.Val669Gly). The in-silico predictions of the variant were probably damaging by PolyPhen-2 (HumDiv) and damaging by SIFT, LRT and MutationTaster2. The phenotype is still largely unknown for this mutation. Her parents and siblings couldn't be tested due to cost issues.

Due to the reported associations of STAT5B mutation with immunodeficiency and GHI, we evaluated the patient for the same. Her primary immunodeficiency evaluation suggested CD8 cell deficiency and raised IgG. (Table 1). On hormonal evaluation, normal baseline and stimulated Growth hormone (GH) levels while reduced IGF-1 levels were suggestive of GHI (Table 1). Her thyroid function tests revealed hypothyroidism with normal Anti-TPO antibody levels (Table 1). Repeat celiac serology (anti-tTG IgA) was negative.

The child was started on oral steroids (Prednisolone at $1 \mathrm{mg} / \mathrm{kg} /$ day) and hydroxychloroquine (10 $\mathrm{mg} / \mathrm{kg} /$ day) for ILD. She was discharged after 10 days of hospital stay. On follow up after 8 months, 
there was improvement in breathlessness (MRC scale 4 to 2) and cough as well as weight gain. There were no exacerbations in this period. She was continued on low dose steroids $(0.5 \mathrm{mg} / \mathrm{kg}$ every alternate day) and hydroxychloroquine. She was being followed up with pediatric endocrinologist for hypothyroidism and GHI and continued on L-Thyroxine $(50 \mathrm{mcg})$. However, she couldn't be started on any GH replacement therapy due to cost issues.

\section{Discussion}

STAT5B mutation is an uncommon cause of ILD. It has been reported to be associated with GHI, immunodeficiency and autoimmunity $(1,2)$. There are 12 case reports of STAT5B mutation or deficiency till now $(2-4)$. Most of these cases had growth failure secondary to GHI and immunodeficiency due to defects in regulatory $\mathrm{T}$ (Treg) cells. Eczema, thrombocytopenic purpura, and/or autoimmune disease like juvenile idiopathic arthritis, autoimmune thyroiditis or celiac disease were present in almost all cases (2-4). Severe pulmonary disease presenting in early childhood was characteristic feature of most of the cases except in 1 case (5). Similarly, our case also manifested the features of GHI, autoimmunity (celiac disease), immunodeficiency (CD8 cell deficiency) and ILD.

Pulmonary involvement in STAT5B mutation has been hypothesized due to decreased numbers of Treg and attenuated effector $\mathrm{T}$ cell (Teff) function or associated autoimmune phenomenon $(1,2)$. Lung involvement has shown varied manifestations in the form of recurrent pulmonary infections, LIP, progressive pulmonary fibrosis and respiratory failure $(2,4)$. On the basis of clinical and radiological findings, we made the diagnosis of fibrosing ILD in contrast to previously reported findings of LIP. However, we couldn't perform lung biopsy for characterization of ILD.

Most of the previous case reports had lymphopenia with reduced CD4 and CD8 T cells, NK cells, Treg cells and function $(2,3)$. We found reduction in total CD3 and naïve CD8 cells with normal CD4 and NK cells. This could be explained because of variable phenotypic expression of STAT5B mutation. We could not perform functional assays of $\mathrm{T}$ cells because of logistic issues. Though $\mathrm{B}$ cells are rarely affected in this disorder, these patients have been reported to have hypergammaglobulinemia similar to our case, low IgM memory B-cells, and increased switched memory B-cell counts (6).

Growth failure in STAT5B mutation has been associated with normal baseline and stimulated GH levels and reduced IGF-1 levels indicating GHI as its cause (1). These patients usually do not respond to recombinant human GH administration (2). Our case did not receive such therapy.

The novel mutation p.Val669Gly in STAT5B gene in our case seems to selectively affecting respiratory system and growth more than leading to immune dysregulation as compared to previous reports as the autoimmunity was less well established in our case.

This case highlights STAT5B mutation as a novel cause of ILD in children. This condition may be thought of in any child with unexplained pulmonary manifestations or childhood ILD of unknown etiology associated GHI, autoimmune conditions and immunodeficiency particularly lymphopenia.

\section{References}

1. Hwa V. STAT5B deficiency: Impacts on human growth and immunity. Growth Horm IGF Res. 2016 Jun;28:16-20.

2. Nadeau K, Hwa V, Rosenfeld RG. STAT5b Deficiency: An Unsuspected Cause of Growth Failure, Immunodeficiency, and Severe Pulmonary Disease. J Pediatr. 2011 May;158(5):701-8.

3. Acres MJ, Gothe F, Grainger A, Skelton AJ, Swan DJ, Willet JDP, et al. Signal transducer and activator of transcription 5B deficiency due to a novel missense mutation in the coiled-coil domain. J Allergy Clin Immunol. 2019 Jan;143(1):413-416.e4.

4. Foley CL, Al Remeithi SS, Towe CT, Dauber A, Backeljauw PF, Tyzinski L, et al. Developmental Adaptive Immune Defects Associated with STAT5B Deficiency in Three Young Siblings. J Clin Immunol. 2021 
Jan;41(1):136-46.

5. Scaglia PA, Martínez AS, Feigerlová E, Bezrodnik L, Gaillard MI, Di Giovanni D, et al. A novel missense mutation in the SH2 domain of the STAT5B gene results in a transcriptionally inactive STAT5b associated with severe IGF-I deficiency, immune dysfunction, and lack of pulmonary disease. J Clin Endocrinol Metab. 2012 May;97(5):E830-839.

6. Bezrodnik L, Di Giovanni D, Caldirola MS, Azcoiti ME, Torgerson T, Gaillard MI. Long-Term Follow-up of STAT5B Deficiency in Three Argentinian Patients: Clinical and Immunological Features. J Clin Immunol. 2015 Apr;35(3):264-72.

\section{Hosted file}

Table 1.docx available at https://authorea.com/users/436451/articles/538753-interstitiallung-disease-in-an-adolescent-child-associated-with-a-novel-stat5b-mutation-a-casereport

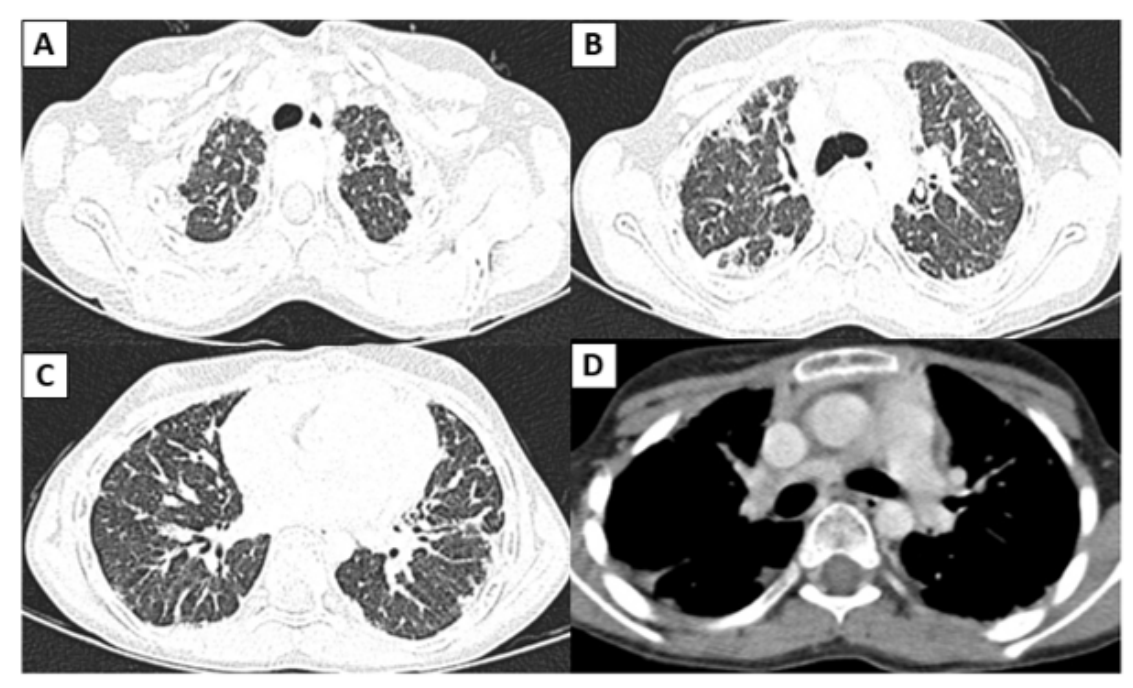

